\title{
A Splitting Method for the Degasperis-Procesi Equation Using an Optimized WENO Scheme and the Fourier Pseudospectral Method
}

\author{
Yunrui Guo ${ }^{1}$, Wenjing Yang ${ }^{1, *}$, Hong Zhang ${ }^{2}$, Ji Wang ${ }^{1}$ \\ and Songhe Song ${ }^{3}$ \\ 1 State Key Laboratory of High Performance Computing, College of Computer, National \\ University of Defense Technology, Changsha 410073, China \\ 2 Department of Mathematics, Utrecht University, The Netherlands P.O.Box 80.010, \\ 3508TA, Utrecht \\ ${ }^{3}$ Department of Mathematics, College of Sciences, National University of Defense \\ Technology, Changsha 410073, China
}

Received 16 February 2018; Accepted (in revised version) 19 July 2018

\begin{abstract}
The Degasperis-Procesi (DP) equation is split into a system of a hyperbolic equation and an elliptic equation. For the hyperbolic equation, we use an optimized finite difference weighted essentially non-oscillatory (OWENO) scheme. New smoothness measurement is presented to approximate the typical shockpeakon structure in the solution to the DP equation, which evidently reduces the dissipation arising from discontinuities simultaneously removing nonphysical oscillations. For the elliptic equation, the Fourier pseudospectral method (FPM) is employed to discretize the high order derivative. Due to the combination of the WENO reconstruction and FPM, the splitting method shows an excellent performance in capturing the formation and propagation of shockpeakon solutions. The numerical simulations for different solutions of the DP equation are conducted to illustrate the high accuracy and capability of the method.
\end{abstract}

AMS subject classifications: 65M10, 78A48

Key words: Degasperis-Procesi equation, discontinuous solution, weighted essentially nonoscillatory method, pseudospectral method.

\section{Introduction}

In this paper, we consider the Degasperis-Procesi equation

$$
u_{t}+3 \kappa^{3} u_{x}-u_{x x t}+4 f(u)_{x}=f(u)_{x x x}
$$

${ }^{*}$ Corresponding author.

Email: yunruiguo@nudt.edu.cn (Y. R. Guo) 
where $u(x, t)$ is a real function and $f(u)=u^{2} / 2$. This equation is an approximate model of shallow water wave propagation in small amplitude and long wavelength regime. It was first found by Degasperis and Procesi when they were studying the asymptotic integrability to the third-order dispersive equation [1]

$$
u_{t}-\alpha^{2} u_{x x t}+\gamma u_{x x x}+c_{0} u_{x}=\left(c_{1} u^{2}+c_{2} u_{x}^{2}+c_{3} u u_{x x}\right)_{x}
$$

with six real constants $c_{0}, c_{1}, c_{2}, c_{3}, \gamma, \alpha \in \mathbb{R}$, for which only three of them satisfy the integrability condition, namely, the Korteweg-de Vries (KdV) equation $\left(\alpha=c_{2}=c_{3}=0\right)$, the Camassa-Holm $(\mathrm{CH})$ equation $\left(c_{1}=-\frac{3 c_{3}}{2 \alpha^{2}}, c_{2}=\frac{c_{3}}{2}\right)$, and the DP equation (1.1). The KdV equation, as the simplest model, has been studied in detail $[2,3]$. While the DP equation is more complicated, because of the existence of the mixed derivative term $u_{x x t}$ and the nonlinear dispersion terms $u u_{x x x}$ and $u_{x} u_{x x}$.

Degasperis proved the integrability of the DP equation by constructing a Lax pair and a bi-Hamiltonian structure [4]. It was related to the AKNS shallow water wave equation by a hodograph transformation [5]. Based on above results, Matsuno obtained the multisoliton solutions of the DP equation for the case $\kappa \neq 0$ [6]. Furthermore, Lundmark and Szmigielski found the explicit form of multipeakon solutions for $\kappa=0$ by solving an inverse scattering problem of a discrete cubic string [7-9]. Additionally, the peakon solutions for these two equations are orbitally stable [10].

One of the important features of the DP equation $(\kappa=0)$ is that it has not only a peaked solution $u(x, t)=c e^{-|x-c t|}[4]$, but also a shock wave solution of the form [11,12]

$$
u(x, t)=c e^{-|x-c t|}+\frac{s}{t s+1} \operatorname{sign}(x-c t) e^{-|x-c t|},
$$

where $c, s(s>0)$ are constants. Moreover, the DP equation possesses a periodic shock wave solution [13] given by

$$
u(x, t)= \begin{cases}\left(\frac{\cosh \left(\frac{1}{2}\right)}{\sinh \left(\frac{1}{2}\right)} t+c\right)^{-1} \frac{\sinh \left(x-|x|-\frac{1}{2}\right)}{\sinh \frac{1}{2}}, & x \in \mathbb{R} \backslash \mathbb{Z}, c>0, \\ 0, & x \in \mathbb{Z} .\end{cases}
$$

Lundmark further extended the multipeakon solution of the DP equation to the multishockpeakon solution [11]

$$
u(x, t)=\sum_{i=1}^{n} m_{i}(t) e^{-\left|x-x_{i}(t)\right|}+\sum_{i=1}^{n} s_{i}(t) \operatorname{sign}\left(x-x_{i}\right) e^{-\left|x-x_{i}(t)\right|},
$$

where $m_{i}(t), x_{i}(t)$ and $s_{i}(t)$ represent the momentum, position and strength of the $i$-th shockpeakon, respectively. It was proved that (1.5) is a weak solution of the DP equation if and only if $m_{i}(t), x_{i}(t)$ and $s_{i}(t)$ satisfy an ODE system. Inspired by the existence of these discontinuous solutions, Coclite et al. developd a well-posedness theory which depends on some functional spaces containing discontinuous functions [11]. They proved 
the existence and uniqueness for entropy weak solutions belonging to the class $L^{1} \cap B V$ and extended the conclusion to a class of generalized DP equations. Furthermore, Lundmark found some explicit shock solutions, i.e., entropy weak solutions to the DP equation [11].

In the last decade, several numerical methods have been proposed for the DP equation. Coclite, Karlsen and Risebro constructed a set of splitting approximations and proved that they converged to entropy weak solutions [14]. Feng and Liu developed another different operator splitting method for the DP equation [7], which was based on a second order TVD method and a linearized implicit finite difference method. However, the spatial accuracies of all above methods are no higher than second order. In order to preserve the Hamiltonian invariants of the DP equation, conservative finite difference methods for continuous solutions were first investigated by Miyatake and Matsuo [15]. Later $\mathrm{Yu}$ et al. proposed a compact finite difference method with symplectic implicit Runge-Kutta integration [16]. Xu and Shu developed and tested high order local discontinuous Galerkin methods for the DP equation, and showed the $L^{2}$ stability for general solutions [17]. Moreover, the Fourier spectral methods with the Gegenbauer postprocessing was adopted to the discontinuous solutions [18], for the purpose of avoiding spurious oscillations nearby the contact discontinuities or strong shocks. Recently, Song et al. proposed a modified structure-preserving method for the DP equation, but it failed to approximate shocks [19].

The WENO methodologies [20,21] are high order numerical simulations for PDEs charactered with discontinuities, sharp gradient regions and other complex solution structures. Xia and $\mathrm{Xu}$ first applied WENO schemes to solve the DP equation $(\kappa=0)$ by decomposing it into a hyperbolic equation and an elliptic equation [22]. This decomposition reflects the mechanism of shock formation in the DP equation to some extent. In our work, the method is based on this decomposition as well. It is worth noting that the solution generally contains some critical points (points of zero derivatives of the flux function) and shockpeakons, comprising a peakon and a shock (i.e., the maximum or minimum generally emerges at a discontinuity). Nonetheless, the classical choice of smoothness indicators in WENO schemes (known as WENO-JS) is inclined to loss accuracy at critical points [23] and amplify dissipations at shockpeakons, resulting from excessively small weights assigned to substencils appearing high gradients. To fix that, a mapping function which corrected the weights was proposed, resulting in WENO-M scheme. On top of fixing the accuracy issue, WENO-M was also the first scheme to show significant improvement on the quality of the solution close to shocks and high gradients. As a drawback, the proposed mapping reveals to be computationally expensive. Alternatively, Borges et al. introduced [24] another new scheme, dubbed as WENO-Z, which achieved superior results with almost the same computational effort of the WENO-JS scheme. This scheme attains much better resolution at the smooth parts of the solution, while keeping the same numerical stability as WENO-JS at shocks and discontinuities. Also, the analogous improvements on smoothness indicators have been present in [25-27] generating the same effect as WENO-Z. 
Our purpose is to extend the WENO reconstructions to the general DP equation using the finite difference discretization. We are interested in a new smoothness measurement, which was studied recently to optimize the fifth order WENO scheme for capturing shock waves [28]. Nevertheless, the original proof, supporting its priority than WENO-JS, is not rigorous enough. Thus a complete theoretical analysis is provided in this paper. Furthermore, we compare our scheme with WENO-Z when approximating shockpeakons. And we discretize the linear dispersion term by FPM instead of linear finite difference methods (LFD) used by $\mathrm{Xu}$ [22]. This method is proved to be effective for periodic initial value problems with constant coefficients, and has been successfully applied to model wave propagation [29].

The rest of the paper is organized as follows. In Section 2, preliminaries about the DP equation and its splitting technique are recalled. Section 3 emphasizes on the construction of the OWENO finite difference scheme for the DP equation. The novel smoothness technique is introduced in detail. In Section 4, the Fourier pseudospectral method is presented for the discretization of the dispersion term. In Section 5, the proposed method is tested on a number of numerical problems. Concluding remarks are given in Section 6.

\section{Preliminary about the DP equation}

We are interested in the DP equation (1.1) with initial condition $u(x, 0)=u_{0}(x)$ in the interval $[-L, L]$, and assume that the solution satisfies the periodic boundary condition. Using the inverse operator $I-\partial_{x x}$, we can split the DP equation into a simple hyperbolicelliptic system [22]

$$
\begin{aligned}
& u_{t}+f(u)_{x}+p=0, \\
& p-p_{x x}=3 f(u)_{x}+3 \kappa^{3} u_{x}
\end{aligned}
$$

for which two useful conservation laws

$$
I_{1}(u)=\int_{R} u d x, \quad I_{2}(u)=\int_{R} u^{3} d x,
$$

are important approaches in testing the preserving ability of the proposed method.

To solve (2.1) numerically, a standard solution procedure starts with the spatial discretization of the equation and then does the time integration. Consider a uniform grid defined by the points

$$
-L=x_{0}<x_{1}<\cdots<x_{N}=L,
$$

which are called cell centers with cell nodes given by $x_{i+\frac{1}{2}}=\frac{h}{2}+x_{i}$, here $h$ is the uniform grid step. The discretized grid functions are denoted by $u_{h}=\left\{u_{i}\right\}_{i=0}^{N-1}$ and $p_{h}=\left\{p_{i}\right\}_{i=0}^{N-1}$ separately. Thus the semi-discrete formulation for the DP equation can be given by

$$
\left\{\begin{array}{l}
\partial_{t} u_{h}+D f\left(u_{h}\right)+p_{h}=0 \\
p_{h}-\Delta p_{h}=3 D f\left(u_{h}\right)+3 \kappa^{3} D u_{h}
\end{array}\right.
$$

where the differential operators $D$ and $\Delta$ indicate the approximations of $\partial_{x}$ and $\partial_{x x}^{2}$. 


\section{High order optimized finite difference WENO method}

In this subsection, we present the fifth order optimized WENO reconstruction (OWENO5) for the hyperbolic equation (2.1a).

The spatial finite difference discretization of Eq. (2.1a) can be written as

$$
\frac{d}{d t} u_{i}+\left.\frac{\partial f}{\partial x}\right|_{x_{i}}+p_{i}=0, \quad i=0,1, \cdots, N-1
$$

For the construction of flux differences across uniformly-spaced cells, a conservative finite difference formulation for the DP equation requires high order consistent numerical fluxes at the cell nodes. Thus we implicitly define the numerical flux function $g(x)$ as

$$
f(x)=\frac{1}{h} \int_{x-\frac{h}{2}}^{x+\frac{h}{2}} g(\xi) d \xi
$$

such that the spatial derivative in Eq. (3.1) is exactly approximated by the following conservative finite difference method

$$
\frac{d}{d t} u_{i}+\frac{1}{h}\left(g_{i+\frac{1}{2}}-g_{i-\frac{1}{2}}\right)+p_{i}=0
$$

where $g_{i+\frac{1}{2}}=g\left(x_{i+\frac{1}{2}}\right)$.

The classical $(2 r-1)$-order WENO method uses a $(2 r-1)$-point global stencil, which is subdivided into $r$ substencils $S_{0}, S_{1}, \cdots, S_{r-1}$ with each substencil containing $r$ grid points. With the smooth flux splitting $f(u)=f^{+}(u)+f^{-}(u)$, we can obtain $(2 r-1)$-order polynomial interpolations to $g_{i \pm \frac{1}{2}}$ and denote them as $\hat{f}_{i \pm \frac{1}{2}}$, where $\frac{d f^{+}(u)}{d u} \geq 0, \frac{d f^{-}(u)}{d u} \leq 0$. Consequently, the conservative finite difference formula for $f_{x}$ can be given by

$$
\left(D f\left(u_{h}\right)\right)_{i}=\frac{1}{h}\left(\hat{f}_{i+\frac{1}{2}}-\hat{f}_{i-\frac{1}{2}}\right), \quad\left(D u_{h}\right)_{i}=\frac{1}{h}\left(\hat{u}_{i+\frac{1}{2}}-\hat{u}_{i-\frac{1}{2}}\right) .
$$

In this paper, the Lax-Friedrichs splitting technique is applied as:

$$
f^{ \pm}(u)=\frac{1}{2}(f(u) \pm \alpha u), \quad \alpha=\max _{u}\left|f^{\prime}(u)\right| .
$$

To be specific, the parameter $\alpha$ is taken by $\max _{u}|u|$ and the constant 1 for $\hat{f}$ and $\hat{u}$, respectively. Thus $\hat{f}_{i \pm \frac{1}{2}}=\hat{f}_{i \pm \frac{1}{2}}^{+}+\hat{f}_{i \pm \frac{1}{2}}^{-}$, both $\hat{f}_{i \pm \frac{1}{2}}^{+}$and $\hat{f}_{i \pm \frac{1}{2}}^{-}$are built through the convex combination of the interpolated values $\hat{f}_{k}^{+}\left(x_{i \pm \frac{1}{2}}\right)$ and $\hat{f}_{k}^{-}\left(x_{i \pm \frac{1}{2}}\right)$. For simplicity, we only work out the reconstruction of $\hat{f}_{i \pm \frac{1}{2}}^{+}$:

$$
\hat{f}_{i \pm \frac{1}{2}}^{+}=\sum_{k=0}^{r-1} \omega_{k} \hat{f}_{k}\left(x_{i \pm \frac{1}{2}}\right)
$$


where $\hat{f}_{k}(x)$ is the $r$-th degree polynomial defined on each of the substencils $S_{k}$,

$$
\hat{f}_{k}\left(x_{i+\frac{1}{2}}\right)=\sum_{j=0}^{r-1} c_{k j} f_{i-k+j}, \quad i=0, \cdots, N .
$$

The Lagrangian interpolation coefficients $c_{k j}$ depend on the parameters $k=0, \cdots, r-1$.

The weights $\omega_{k}$ are defined as

$$
\omega_{k}=\frac{\alpha_{k}}{\sum_{l=0}^{r-1} \alpha_{l}}, \quad \alpha_{k}=\frac{d_{k}}{\left(\beta_{k}+\epsilon\right)^{2}}, \quad \beta_{k}=\sum_{l=1}^{r-1} h^{2 l-1} \int_{x_{i-\frac{1}{2}}}^{x_{i+\frac{1}{2}}}\left(\frac{d^{l}}{d x^{l}} \hat{f}_{k}(x)\right)^{2} d x,
$$

where $\epsilon$ is a positive real number to prevent the denominator from being zero. We take it as $10^{-6}$ in numerical experiments. The coefficients $d_{0}, d_{1}, \cdots, d_{r-1}$ are ideal weights [20].

\subsection{A new smoothness measurement}

For the classical smoothness measurement (3.5), the indicators $\beta_{k}$ are small enough on smooth parts of the solution so that $\omega_{k}$ approximate the ideal weights $d_{k}$ very well. However, if one substencil $S_{k}$ contains discontinuities, then

$$
\beta_{k}=\mathcal{O}(1)
$$

Consequently the corresponding weight $\omega_{k}$ approximating zero produces excessive dissipation and weakens the ability to capture shocks. In this subsection, we present a novel approach to measure the smoothness of numerical solutions on a stencil [28].

Firstly, we introduce conjugate smoothness indicators:

$$
\bar{\beta}_{k}=\sum_{l=0, l \neq k}^{r-1} \beta_{k}, \quad k=0,1, \cdots, r-1 .
$$

Then, the new indicators

$$
\beta_{k}^{E}=\frac{\epsilon+\bar{\beta}_{k}}{\epsilon+\beta_{k}}, \quad k=0,1, \cdots, r-1,
$$

depend on both $\bar{\beta}_{k}$ and $\beta_{k}$. Thus the optimized nonlinear weights $\omega_{k}^{E}$ can be formulated by

$$
\alpha_{k}=d_{k} \beta_{k}^{E}, \quad \omega_{k}^{E}=\frac{\alpha_{k}}{\sum_{l=0}^{r-1} \alpha_{l}}, \quad k=0,1, \cdots, r-1 .
$$

The new construction of nonlinear weights considers the smoothness interaction among various substencils. In smooth regions, the optimized weights $\omega_{k}^{E}$ are closer to the ideal weights than the classical weights $\omega_{k}$. Therefore, the former reconstruction approximates the exact solution better. In discontinuous regions, $\omega_{k}^{E}$ from "discontinuous" substencils 
are amplified moderately, which efficiently reduce the dissipation and improve the accuracy of discontinuities.

Next, we present a simple analysis and a comparison of both the smoothness techniques theoretically. For convenience, $\epsilon$ is taken as zero. We select the weight of $S_{0}$ as an example to illustrate the case of $r=3$.

In smooth regions:

$$
\begin{aligned}
\omega_{0} & =\frac{d_{0}}{d_{0}+d_{1}\left(\frac{\beta_{0}}{\beta_{1}}\right)^{2}+d_{2}\left(\frac{\beta_{0}}{\beta_{2}}\right)^{2}}, \\
\omega_{0}^{E} & =\frac{d_{0}}{d_{0}+d_{1}\left(\frac{\beta_{0}}{\beta_{1}+\beta_{2}}\right)\left(\frac{\beta_{0}+\beta_{2}}{\beta_{1}}\right)+d_{2}\left(\frac{\beta_{0}}{\beta_{1}+\beta_{2}}\right)\left(\frac{\beta_{0}+\beta_{1}}{\beta_{2}}\right)} \\
& =\frac{d_{0}}{d_{0}+d_{1}\left(\frac{\beta_{0}}{\beta_{1}}\right)\left(\frac{\beta_{0}+\beta_{2}}{\beta_{1}+\beta_{2}}\right)+d_{2}\left(\frac{\beta_{0}}{\beta_{2}}\right)\left(\frac{\beta_{0}+\beta_{1}}{\beta_{1}+\beta_{2}}\right)} .
\end{aligned}
$$

We are interested in the parameters of $d_{1}$ and $d_{2}$ in the denominators of Eq. (3.9a) and Eq. (3.9b):

Case 1. If $\beta_{0} \leq \beta_{1}, \beta_{2}$, then

$$
\begin{array}{ll}
\frac{\beta_{0}}{\beta_{1}} \leq 1, & \left(\frac{\beta_{0}}{\beta_{1}}\right)^{2} \leq\left(\frac{\beta_{0}}{\beta_{1}}\right)\left(\frac{\beta_{0}+\beta_{2}}{\beta_{1}+\beta_{2}}\right) \leq 1, \\
\frac{\beta_{0}}{\beta_{2}} \leq 1, & \left(\frac{\beta_{0}}{\beta_{2}}\right)^{2} \leq\left(\frac{\beta_{0}}{\beta_{2}}\right)\left(\frac{\beta_{0}+\beta_{1}}{\beta_{1}+\beta_{2}}\right) \leq 1 .
\end{array}
$$

Obviously, the denominator of $\omega_{0}^{E}$ is closer to 1 , so $\omega_{0}^{E}$ is closer to the ideal weight $d_{0}$ than $\omega_{0}$.

Case 2. If $\beta_{0}>\beta_{1}, \beta_{2}$, then

$$
\begin{array}{ll}
\frac{\beta_{0}}{\beta_{1}}>1, & \left(\frac{\beta_{0}}{\beta_{1}}\right)^{2}>\left(\frac{\beta_{0}}{\beta_{1}}\right)\left(\frac{\beta_{0}+\beta_{2}}{\beta_{1}+\beta_{2}}\right)>1, \\
\frac{\beta_{0}}{\beta_{2}}>1, & \left(\frac{\beta_{0}}{\beta_{2}}\right)^{2}>\left(\frac{\beta_{0}}{\beta_{2}}\right)\left(\frac{\beta_{0}+\beta_{1}}{\beta_{1}+\beta_{2}}\right)>1 .
\end{array}
$$

The conclusion is same as Case 1 .

Case 3. For $\beta_{1}<\beta_{0} \leq \beta_{2}$ (similar to $\beta_{2}<\beta_{0}<\beta_{1}$ ), we can discuss both $\beta_{1}$ and $\beta_{2}$ just like $\beta_{0}$ in Case 2 and Case 1 separately. It demonstrates that $\omega_{1}^{E}, \omega_{2}^{E}$ are closer to $d_{1}, d_{2}$ than $\omega_{1}$, $\omega_{2}$. According to $\omega_{0}^{E}+\omega_{1}^{E}+\omega_{2}^{E}=1$, we can infer the same result holding for $\omega_{0}^{E}$ as well.

In discontinuous regions:

Assume that $S_{0}$ contains a discontinuity, $S_{1}$ and $S_{2}$ are smooth, i.e., $\beta_{0}>\beta_{1}$ and $\beta_{0}>\beta_{2}$. Then we have

$$
\frac{\omega_{0}^{E}}{\omega_{1}^{E}}=\frac{d_{0}}{d_{1}}\left(\frac{\beta_{1}}{\beta_{0}}\right)\left(\frac{\beta_{1}+\beta_{2}}{\beta_{0}+\beta_{2}}\right)>\frac{d_{0}}{d_{1}}\left(\frac{\beta_{1}}{\beta_{0}}\right)^{2}=\frac{\omega_{0}}{\omega_{1}} .
$$


Apparently the value of $\omega_{0}^{E}$ increases, which also holds for $\omega_{0}^{E} / \omega_{2}^{E}$.

In conclusion, the new weights $\omega_{k}^{E}$ in smooth parts approximate the ideal weights $d_{k}$ better, and the weights $\omega_{0}^{E}$ in discontinuous parts are assigned relatively large. This distinguishing feature has a great advantage over capturing shockpeakon solutions.

\section{The Fourier Pseudospectral method for the elliptic equation}

In this section, we consider the discretization of the elliptic equation. The FPM has remarkable conservation properties and high spatial accuracy [30]. Unlike the spectral method on Fourier space, the method is on real space and does not need the passage between Fourier space and physical space. So the computation costs less CPU time. It has been widely used to solve Hamiltonian PDEs, such as the Zakharov-Kuznetsov equation, the Kadomtsev-Petviashvili equation [30] and the coupled Schrodinger-KdV equation [31]. In this subsection, we will apply it to Eq. (2.1b) with periodic boundary condition. The discretization is implemented through a trigonometric polynomial at collocation points $\left\{x_{i}\right\}_{i=0}^{N-1}$, where $N$ is an even number, and the period is $2 L$. We approximate $u(x, t)$ by

$$
I_{N} u(x, t)=\sum_{j=0}^{N-1} u_{j} y_{j}(x),
$$

with the $\frac{N}{2}$-degree trigonometric polynomials $y_{j}(x)$ given explicitly by

$$
y_{j}(x)=\frac{1}{N} \sum_{l=-\frac{N}{2}}^{\frac{N}{2}} \frac{1}{C_{l}} e^{i l \mu\left(x-x_{j}\right)},
$$

where $C_{l}=1\left(|l| \neq \frac{N}{2}\right), C_{\frac{N}{2}}=C_{-\frac{N}{2}}=2, \mu=\frac{\pi}{L}$. Substituting Eq. (4.2) into the expression of $I_{N} u(x, t)$, we obtain

$$
I_{N} u(x, t)=\frac{1}{N} \sum_{l=-\frac{N}{2}}^{\frac{N}{2}} \frac{1}{c_{l}} e^{i l \mu x} \sum_{j=0}^{N-1} u_{j} e^{-i l \mu\left(x_{j}\right)}
$$

Then

$$
\begin{aligned}
& I_{N} u\left(x_{i}, t\right)=\frac{1}{N} \sum_{l=-\frac{N}{2}}^{\frac{N}{2}} \frac{1}{c_{l}} e^{i l \mu x_{i}} \sum_{j=0}^{N-1} u_{j} e^{-i l \mu\left(x_{j}\right)}, \\
& u_{i}=I_{N} u\left(x_{i}, t\right), \quad i=0,1, \cdots, N-1 .
\end{aligned}
$$

The values of the derivative $\frac{\partial^{k} I_{N} u\left(x_{i}, t\right)}{\partial x^{k}}$ at the collocation points $x_{j}$ are obtained by

$$
\frac{\partial^{k} I_{N} u\left(x_{i}, t\right)}{\partial x^{k}}=\sum_{j=0}^{N-1} u_{j} \frac{d^{k} y_{n}\left(x_{i}\right)}{d x^{k}}=\left(D_{k} u\right)_{i}, \quad\left(D_{k}\right)_{j, n}=\frac{d^{k} y_{n}\left(x_{j}\right)}{d x^{k}},
$$


where $D_{k}$ represents Fourier pseudospectral differential matrix with the elements

$$
\left(D_{1}\right)_{i, j}= \begin{cases}\frac{1}{2} \mu(-1)^{i+j} \cot \left(\mu \frac{x_{i}-x_{j}}{2}\right), & i \neq j, \\ 0, & i=j .\end{cases}
$$

Therefore, the Fourier pseudospectral discretization for Eq. (2.1) can be written explicitly as:

$$
p_{h}-D_{1}^{2} p_{h}=3 D f\left(u_{h}\right)+3 \kappa^{3} D u_{h}
$$

Remark 4.1. In this paper, we restrict our consideration to the splitting strategy between FPM and various WENO methods only. In fact, the presented splitting framework is also available to general numerical approaches, such as the wavelet collocation method [31] and DG method. For instance, we also test the combination of WENO-Z and the wavelet collocation method in this framework. A good agreement is obtained in Section 5.

\section{Numerical examples}

In this section, we provide several numerical examples to illustrate the accuracy and capability of the proposed method. Considering the total variation bounded property of the DP equation, we employe the third order explicit TVD Runge-Kutta method [20] for time integration. Firstly, the numerical convergence is verified by a single soliton propagation, consisting of a single smooth soliton when $\kappa \neq 0$, and a single peakon when $\mathcal{\kappa}=0$. Then the proposed method is employed to simulate two-peakon solutions

$$
u(x, t)=\sum_{i=1}^{2} c_{i}(t) e^{-\left|x-x_{i}(t)\right|}
$$

including binary peakon-peakon and shockpeakon interactions. Besides, a delicate triple Peakon-Antipeakon-Shockpeakon interaction is resolved to verify the effect of the new smoothness technique. Finally, two types of general initial value problems are provided to verify the resolution and stability of the proposed method.

\subsection{Single soliton solutions}

To test the accuracy of the proposed method, we first solve the DP equation with a smooth solution.

Example 5.1 (Single smooth soliton). When $\kappa \neq 0$ the DP Eq. (1.1) admits smooth $N$ soliton solutions in the parametric form [6], in which a smooth single soliton solution is 


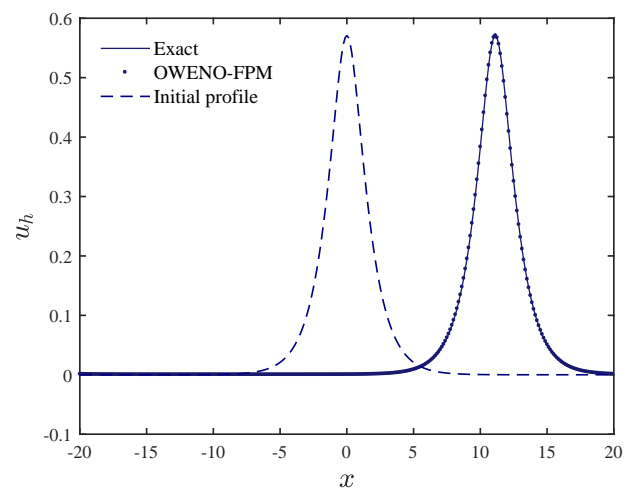

Figure 1: The numerical results computed with $h=0.125$ and $\Delta t=0.0625$ of single soliton solution.

Table 1: Convergence rate for a smooth soliton solution.

\begin{tabular}{||ccccccc||}
\hline$N$ & $L_{\infty}$ error & order & $L_{1}$ error & order & $E_{1}$ & $E_{2}$ \\
\hline 80 & $4.08 \times 10^{-3}$ & - & $2.39 \times 10^{-4}$ & - & $4.95 \times 10^{-7}$ & $1.05 \times 10^{-3}$ \\
160 & $3.04 \times 10^{-4}$ & 3.74 & $1.03 \times 10^{-5}$ & 4.53 & $2.99 \times 10^{-8}$ & $6.14 \times 10^{-5}$ \\
320 & $1.16 \times 10^{-5}$ & 4.71 & $3.30 \times 10^{-7}$ & 4.96 & $1.48 \times 10^{-8}$ & $2.51 \times 10^{-6}$ \\
640 & $4.92 \times 10^{-7}$ & 4.56 & $1.17 \times 10^{-8}$ & 4.82 & $7.48 \times 10^{-9}$ & $9.46 \times 10^{-8}$ \\
\hline
\end{tabular}

of the form

$$
\begin{aligned}
& u(y, t)=\frac{2 \kappa^{3}\left(a_{1}^{2}-1\right)\left(4 a_{1}^{2}-1\right)}{a_{1}\left(\cosh \xi_{1}+2 a_{1}-a_{1}^{-1}\right)}, \\
& x(y, t)=\frac{y}{\kappa}+\ln \left(\frac{\alpha_{1}+1+\left(\alpha_{1}-1\right) e^{\xi_{1}}}{\alpha_{1}-1+\left(\alpha_{1}+1\right) e^{\zeta_{1}}}\right) .
\end{aligned}
$$

Here

$$
\xi_{1}=k_{1}\left(y-\frac{3 \kappa^{4}}{1-\kappa^{2} k_{1}^{2}} t-y_{0}\right), \quad a_{1}=\frac{1}{2} \sqrt{\frac{4-\kappa^{2} k_{1}^{2}}{1-\kappa^{2} k_{1}^{2}}}, \quad \alpha_{1}=\sqrt{\frac{\left(2 a_{1}-1\right)\left(a_{1}+1\right)}{\left(2 a_{1}+1\right)\left(a_{1}-1\right)}} .
$$

We carry out the simulation in a domain $[-20,20]$ with parameters $\kappa=0.511, \kappa k_{1}=0.8$, $y_{0}=0$. The profiles in Fig. 1 are the numerical and the exact solution at $t=10$, which display that the overall solution transition is of high accuracy. Table 1 shows $L_{\infty}, L_{2}$ errors and two conservative quantities $I_{1}, I_{2}$ at $t=1$. . Here $E_{1}=\left|I_{1}-\bar{I}_{1}\right|$ and $E_{2}=\left|I_{2}-\bar{I}_{2}\right|$ indicate the relative errors in $I_{1}$ and $I_{1} ; \bar{I}_{i}$ and $I_{i}(i=1,2)$ stand for the numerical and exact values. Trapezoidal rule is employed for the numerical quadrature of the integrals. The table shows that the proposed scheme can achieve the designed fifth order accuracy.

Example 5.2 (Single peakon solution). Consider the traveling solution

$$
u(x, t)=c e^{-|x-c t|}
$$


Table 2: Comparison of errors between OWENO-FPM and WENO-JS-LFD.

\begin{tabular}{||c|c|ccc||}
\hline Time & Method & $L_{\infty}$ & $E_{1}$ & $E_{2}$ \\
\hline \multirow{2}{*}{$t=2.0$} & FPM & $4.25 \times 10^{-2}$ & $1.13 \times 10^{-8}$ & $1.89 \times 10^{-3}$ \\
& LFD & $4.56 \times 10^{-2}$ & $2.36 \times 10^{-8}$ & $2.20 \times 10^{-3}$ \\
\hline \multirow{2}{*}{$t=4.0$} & FPM & $4.38 \times 10^{-2}$ & $1.08 \times 10^{-7}$ & $3.35 \times 10^{-3}$ \\
& LFD & $4.41 \times 10^{-2}$ & $8.28 \times 10^{-7}$ & $4.34 \times 10^{-3}$ \\
\hline \multirow{2}{*}{$t=6.0$} & FPM & $4.39 \times 10^{-2}$ & $1.51 \times 10^{-6}$ & $4.88 \times 10^{-3}$ \\
& LFD & $4.72 \times 10^{-2}$ & $1.11 \times 10^{-6}$ & $6.75 \times 10^{-3}$ \\
\hline \multirow{2}{*}{$t=8.0$} & FPM & $4.41 \times 10^{-2}$ & $6.14 \times 10^{-6}$ & $6.41 \times 10^{-3}$ \\
& LFD & $4.71 \times 10^{-2}$ & $1.11 \times 10^{-5}$ & $9.23 \times 10^{-3}$ \\
\hline
\end{tabular}

for the DP equation when $\kappa=0$. In our numerical simulation, we choose the traveling speed $c=1.0$ and the computation domain $[-20,20]$ with $N=640$. Table 2 compares the errors of $u_{h}$ and two conservative quantities for different methods at different times. It shows that the proposed method maintains the accuracy and conservative quantities relatively better than WENO-JS-LFD [22], where LFD stands for the linear finite difference method.

\subsection{Peakons and shockpeakons interactions}

Example 5.3 (Two-peakon interaction). In this example, we consider the two-peakon solution for the DP equation $(\kappa=0)$ with the initial condition

$$
u(x, 0)=c_{1} e^{-\left|x-x_{1}\right|}+c_{2} e^{-\left|x-x_{2}\right|},
$$

where the parameters $c_{1}=2.0, c_{2}=1.0, x_{1}=-13.792$ and $x_{2}=-4.0$. In these interactions, the peakon should preserve its shape and velocity before and after encountering a nonlinear interaction with other similar peakon [18]. From Fig. 2, we can observe the precise snapshots at different times during the whole collision process. Furthermore, we continue the interaction to $t=20$. For all methods, the numerical results in Fig. 3 show accurate approximations to the peakon location except for WENO-JS-LFD, but WENOJS-FPM and OWENO-FPM demonstrate relatively better approximations to the peakon amplitude.

Example 5.4 (Shockpeakon solution). The DP equation also admits the shockpeakon solution

$$
u(x, t)=-\frac{1}{t+1} \operatorname{sign}(x) e^{-|x|},
$$

containing a discontinuity at $x=0$ in the domain $[-25,25]$, which always triggers oscillations for general linear schemes. In this example, we investigate, through comparison with WENO-JS and WENO-Z, the behavior of optimized smoothness technique under two coarse grids. Table 3 lists their relative errors and convergence rates when discontinuity occurs. Fig. 4 provides a comparison with the exact solution at $t=3.0$. Obviously, 

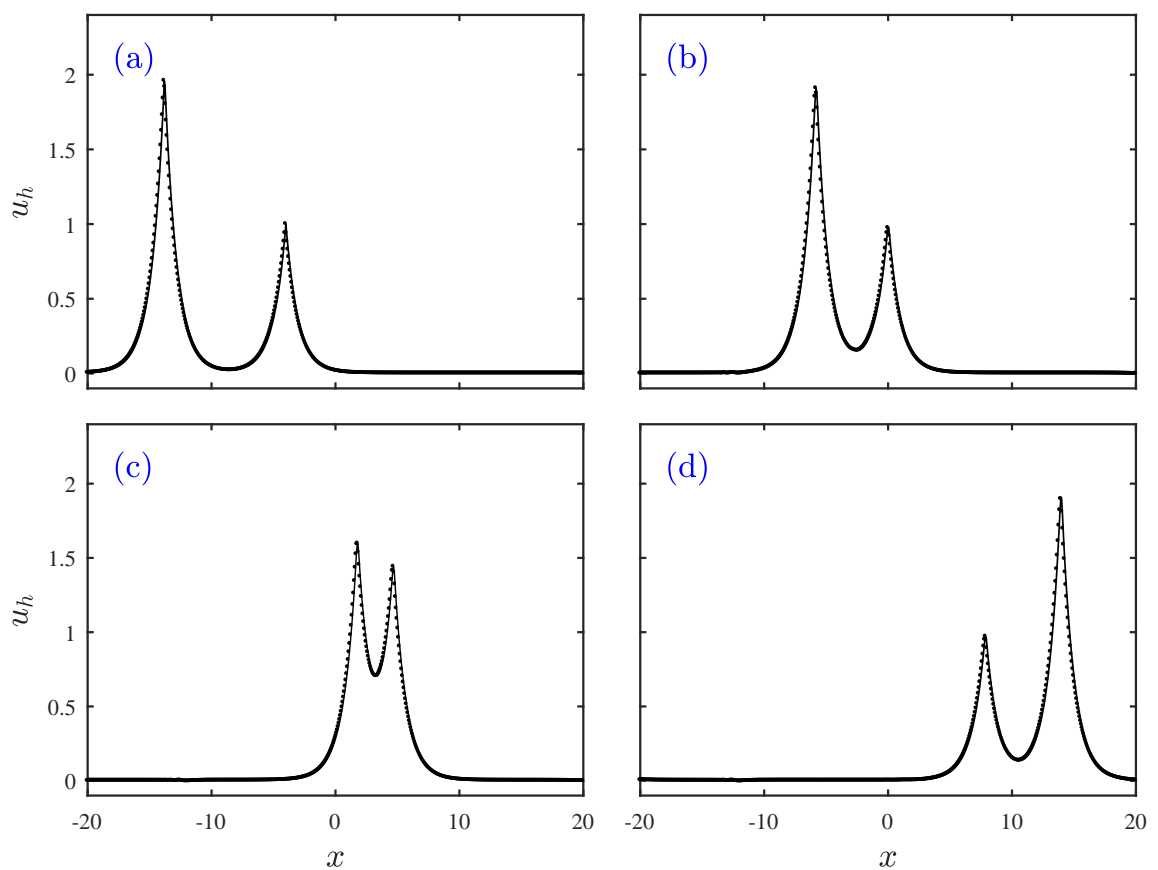

Figure 2: Snapshots for the interaction of two-peakon solutions (a) $t=0$; (b) $t=4$; (c) $t=8$; (d) $t=13$ with $\mathrm{N}=640$ in the domain $[-20,20]$.

Table 3: Comparison of errors for different methods of shockpeakon solution.

\begin{tabular}{||c|c|ccccc||}
\hline Method & Mesh size & $L_{\infty}$ & Order & $L_{1}$ & Order & $E_{1}$ \\
\hline \multirow{2}{*}{ WENO-JS-LFD } & 200 & $4.04 \times 10^{-3}$ & & $1.41 \times 10^{-4}$ & & $1.78 \times 10^{-12}$ \\
& 400 & $5.84 \times 10^{-3}$ & -0.53 & $7.10 \times 10^{-5}$ & 0.99 & $1.94 \times 10^{-11}$ \\
\hline \multirow{2}{*}{ WENO-JS-FPM } & 200 & $3.92 \times 10^{-3}$ & & $1.39 \times 10^{-4}$ & & $1.78 \times 10^{-12}$ \\
& 400 & $5.84 \times 10^{-3}$ & -0.57 & $7.09 \times 10^{-5}$ & 0.97 & $7.60 \times 10^{-13}$ \\
\hline \multirow{2}{*}{ OWENO-FPM } & 200 & $1.28 \times 10^{-2}$ & & $1.51 \times 10^{-4}$ & & $1.76 \times 10^{-12}$ \\
& 400 & $4.36 \times 10^{-3}$ & 1.55 & $1.11 \times 10^{-4}$ & 1.83 & $7.57 \times 10^{-13}$ \\
\hline \multirow{2}{*}{ WENO-Z-FPM } & 200 & $8.54 \times 10^{-2}$ & & $6.14 \times 10^{-4}$ & & $2.49 \times 10^{-12}$ \\
& 400 & $4.54 \times 10^{-2}$ & 0.91 & $1.11 \times 10^{-5}$ & 1.82 & $1.20 \times 10^{-12}$ \\
\hline
\end{tabular}

the resolution of OWENO-FPM is superior than other three methods in the vicinity of the shock.

Example 5.5 (A triple interaction). The triple interaction conducts among peakon, antipeakon and one stationary shockpeakon $[11,14]$, with initial condition

$$
u(x, 0)=e^{-|x+5|}+\operatorname{sign}(x) e^{-|x|}-e^{-|x-5|},
$$

in the computational domain $[-20,20]$. The profiles in Fig. 5 reveal the formation process of multiple shocks accurately. For this example, WENO-Z-FPM have a higher convergence rate than other three methods, as shown in Table 4. 

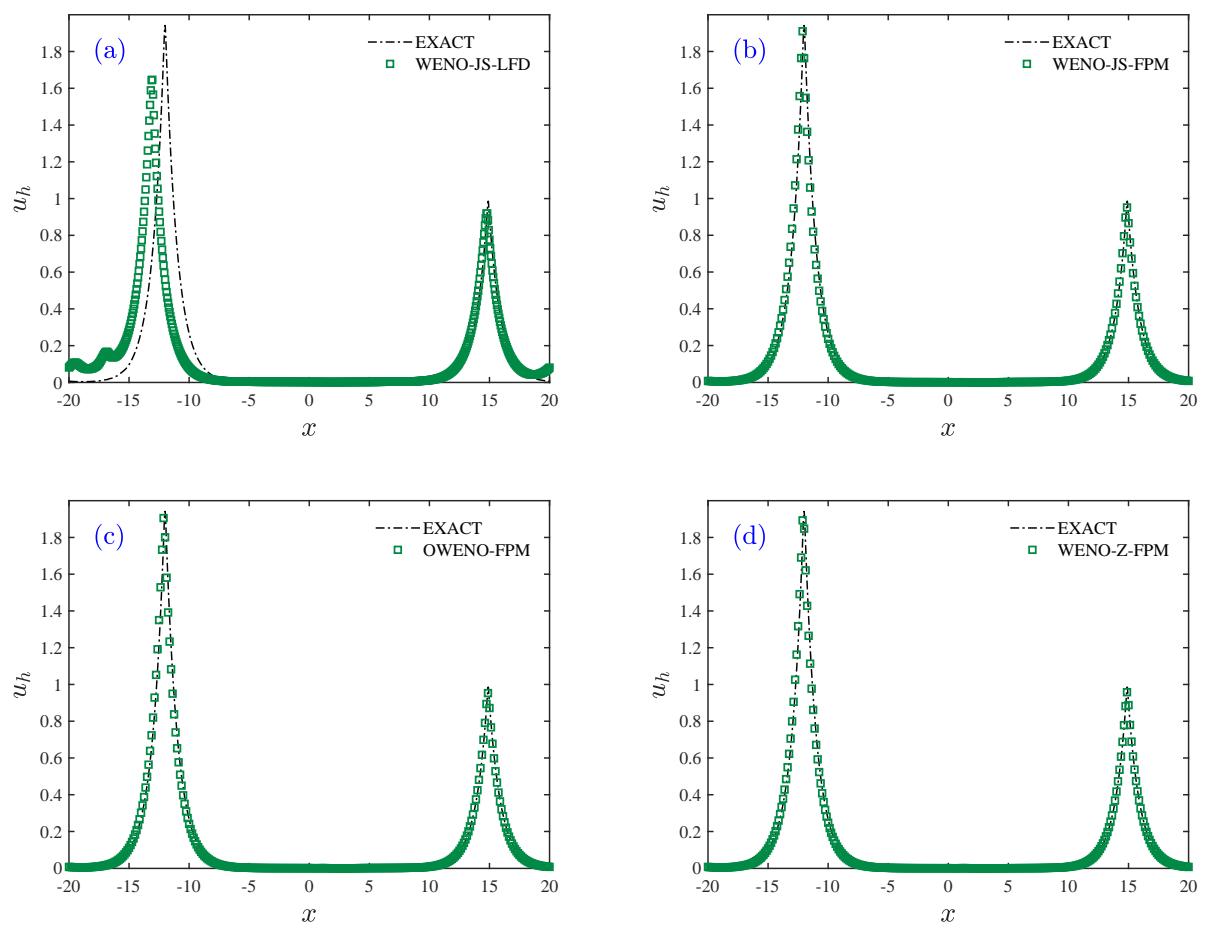

Figure 3: The numerical results with $N=640$ : (a) WENO-JS-LFD; (b) WENO-JS-FPM; (c) OWENO-FPM; (d) WENO-Z-FPM.

Table 4: Comparison of errors for different methods of triple interaction.

\begin{tabular}{||ccccccc||}
\hline Method & $320\left(L_{\infty}\right)$ & $640\left(L_{\infty}\right)$ & Order & $320\left(L_{1}\right)$ & $640\left(L_{1}\right)$ & Order \\
\hline WENO-JS-LFD & $2.694 \times 10^{-1}$ & $1.042 \times 10^{-1}$ & 1.36 & $5.700 \times 10^{-2}$ & $2.145 \times 10^{-2}$ & 1.41 \\
WENO-JS-FPM & $2.695 \times 10^{-1}$ & $1.043 \times 10^{-1}$ & 1.36 & $5.700 \times 10^{-2}$ & $2.145 \times 10^{-2}$ & 1.41 \\
OWENO-FPM & $2.635 \times 10^{-1}$ & $1.009 \times 10^{-1}$ & 1.38 & $5.311 \times 10^{-2}$ & $1.967 \times 10^{-2}$ & 1.43 \\
WENO-Z-FPM & $2.422 \times 10^{-1}$ & $8.951 \times 10^{-2}$ & 1.43 & $4.682 \times 10^{-2}$ & $1.659 \times 10^{-2}$ & 1.50 \\
\hline
\end{tabular}

\subsection{General initial value problems}

Example 5.6 (Shock formation). In this example, we are interested in the shock formation with the smooth initial condition

$$
u(x, 0)=e^{0.5 x^{2}} \sin (\pi x), \quad x \in[-2,2] .
$$

Fig. 6 shows the snapshots during the process of shock formation and transition with $N=512$. It demonstrates that even with the smooth initial condition, shocks appear in finite time. Also, we perform an comparative experiment on coarse grid $N=256$ to test the stability of capturing shock. As shown in snapshot (b) of Fig. 7, the local oscillations appearing at discontinuities spread into the whole space, which can be attributed to the 

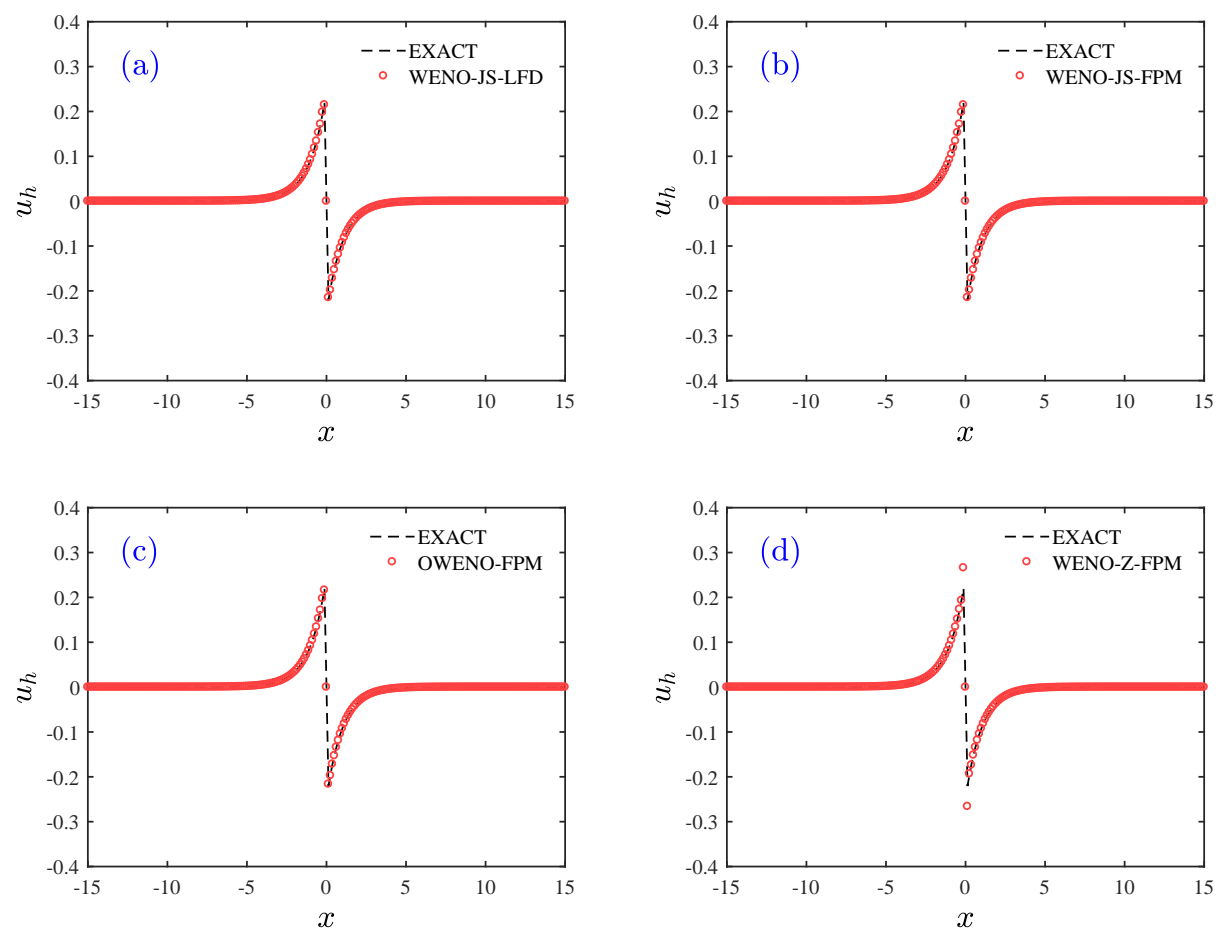

Figure 4: Numerical results of shockpeakon solution at $t=3.0$ with $N=400$.

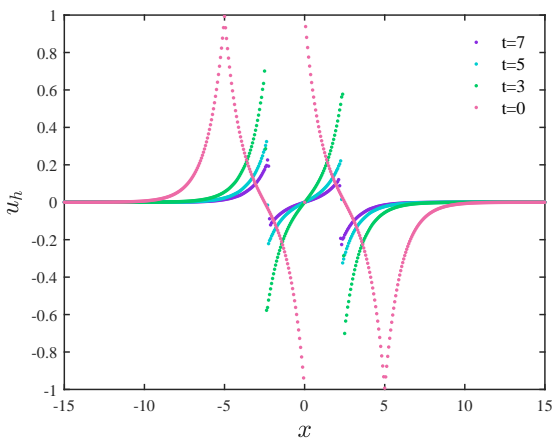

Figure 5: The triple interaction at different times with $N=512$ in the domain $[-20,20]$.

insufficient dissipation. While WENO-JS-LFD is the most dissipative one, OWENO-FPM and WENO-Z-FPM occupy intermediary levels and provide sharper approximations to the shock shape than WENO-JS-LFD.

Example 5.7 (Wave breaking). In the last example, we consider the evolution of the initial condition

$$
u(x, 0)=\operatorname{sech}^{2}\left(d\left(x-x_{0}\right)\right),
$$



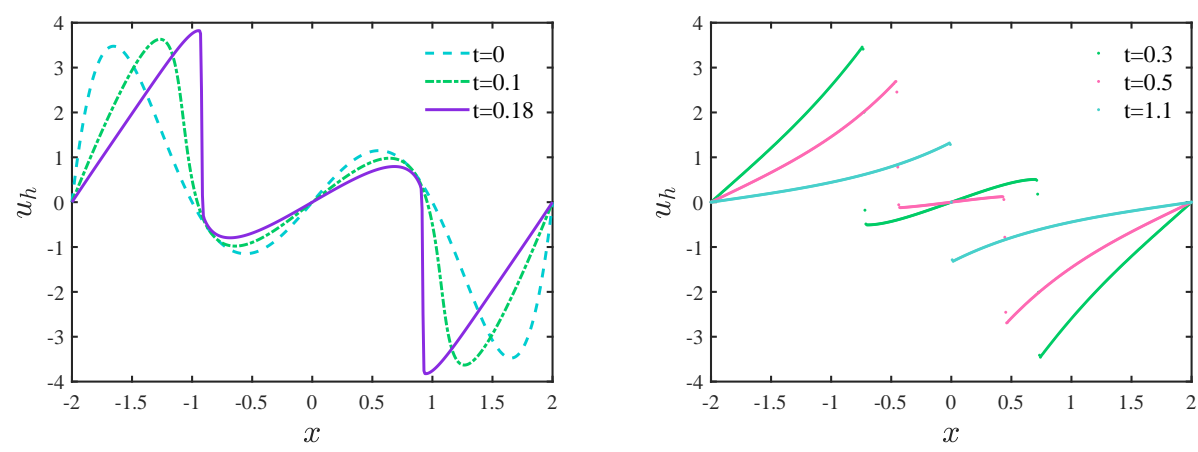

Figure 6: The snapshots for the shock formation at different times.
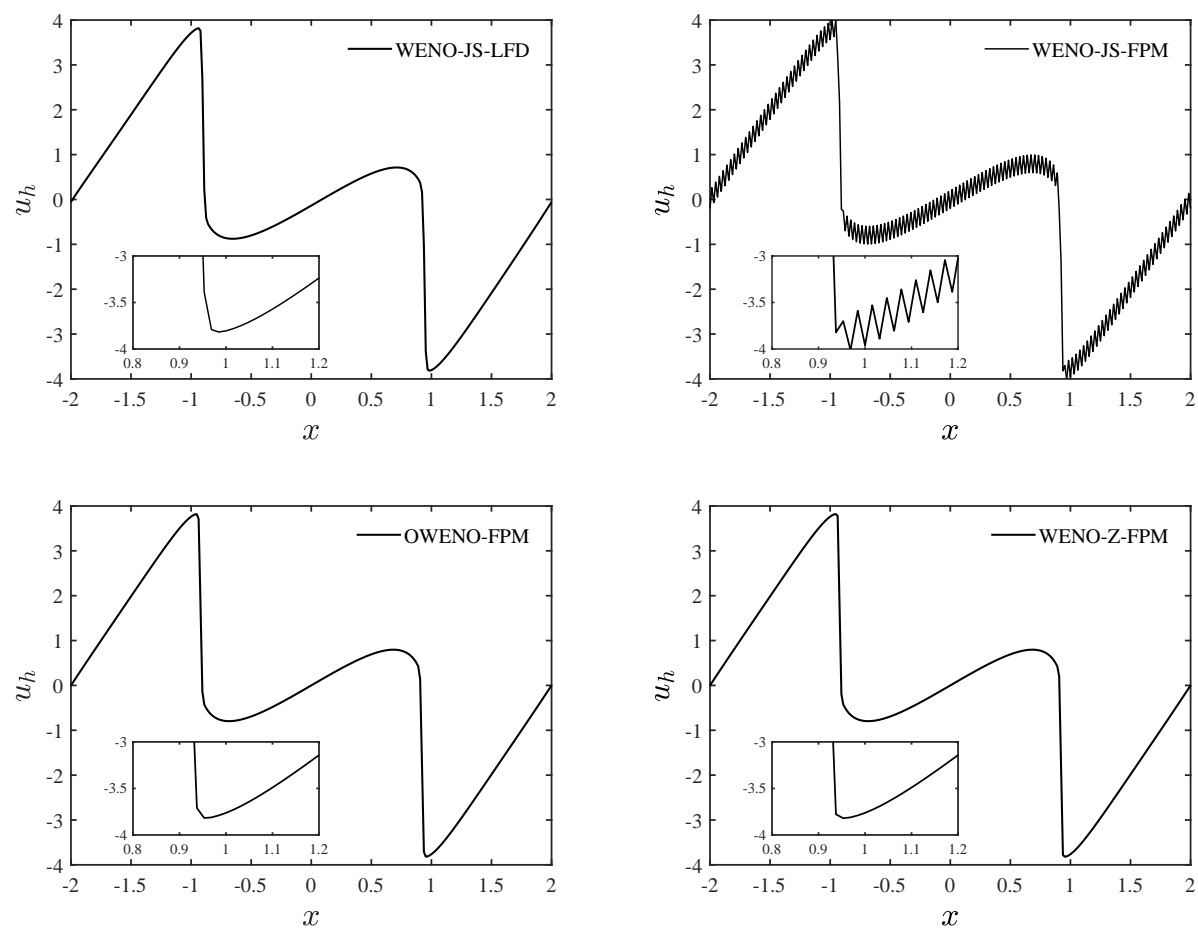

Figure 7: The numerical solutions computed by WENO-JS-FPM, WENO-FPM, OWENO-FPM and WENO-ZFPM with $N=256$ and $C F L=1 / 6$

for $\kappa \neq 0$. The parameters are chosen as $d=0.1, x_{0}=-50$ and the computational domain is $[-100,100]$. The value of $\kappa$ is 0.01 , which implies a very small dispersion term, corresponding to the dispersiveness of the DP equation. The initial profile and the approximate solution at $t=60$ are shown in Fig. 8. This result is similar to the results presented in [7], we can observe that a structured peakon train is generated gradually. Furthermore, we compare the conservative quantities preserved by different methods in Fig. 9. For this 


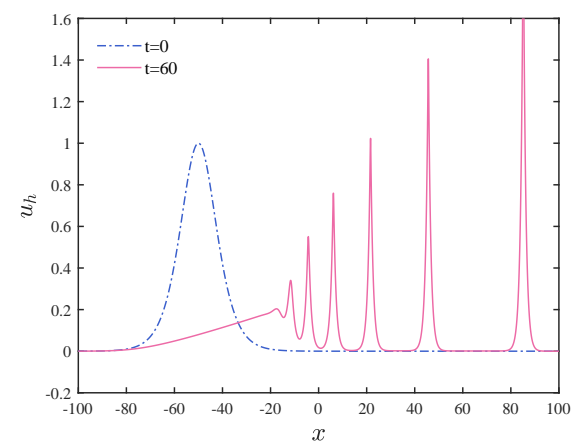

Figure 8: The numerical solution from initial condition with $d=0.1$ and $\kappa=0.01$.
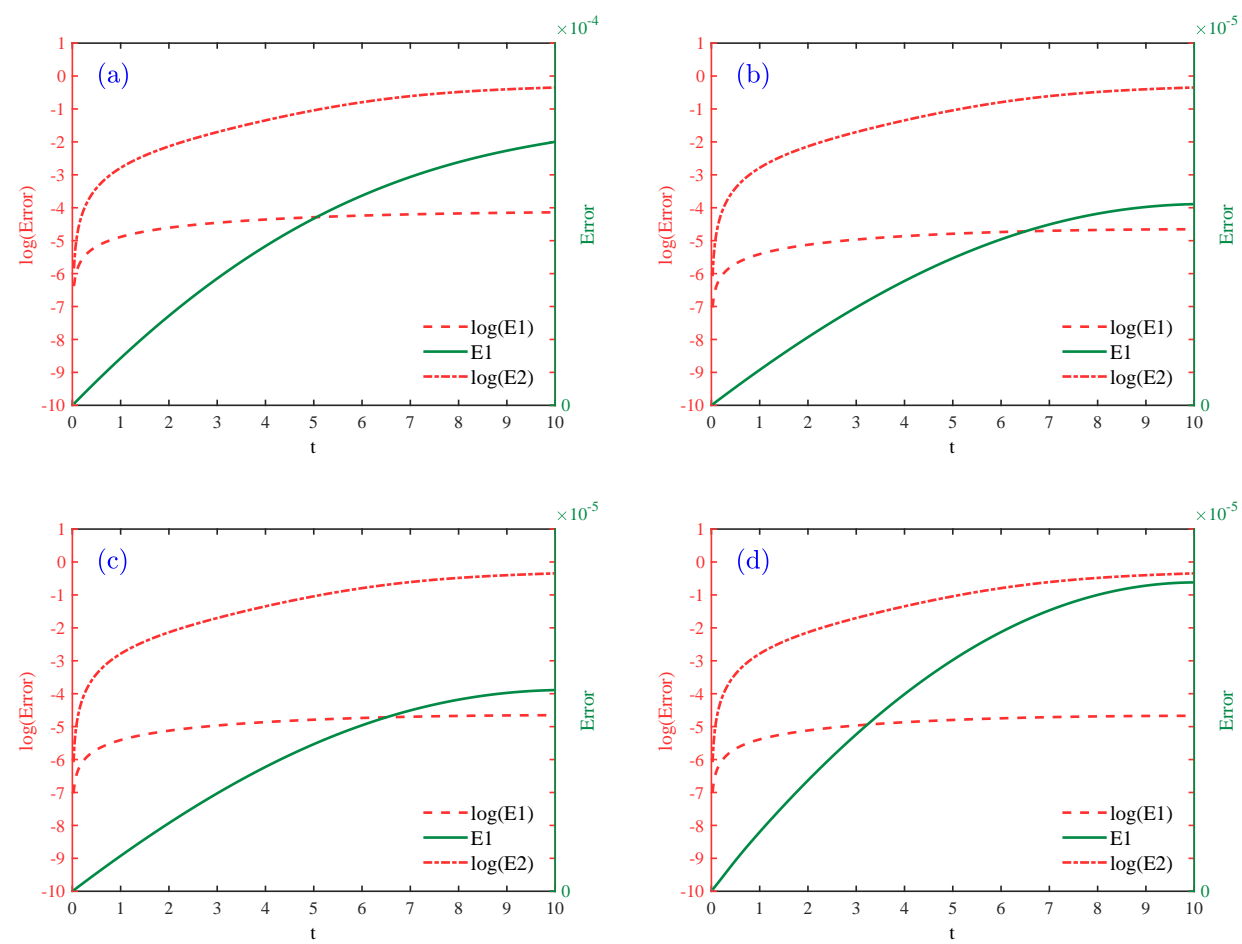

Figure 9: The conservative quantity errors for different methods with $N=256$ and $C F L=1 / 6$.

case, we can see no obvious difference of $I_{2}$ error, but WENO-JS-FPM and OWENO-FPM show smaller $I_{1}$ error than other two methods.

Example 5.8 (Shockpeakon-shockpeakon interaction). To validate the compatibility and flexibility of the splitting framework, we further compute a Shockpeakon-shockpeakon interaction problem using WENO-Z and the wavelet collocation method. The initial con- 

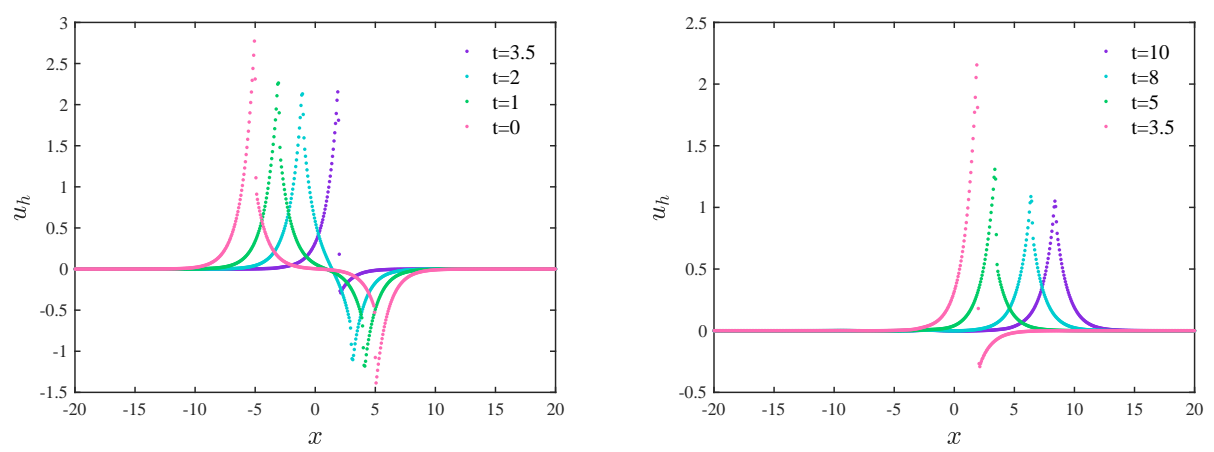

Figure 10: The numerical solutions computed by WENO-Z and the wavelet collocation method with $N=640$ and $C F L=1 / 8$.

dition is set to be

$$
u(x, 0)=\sum_{i=1}^{2} m_{i} e^{-\left|x-x_{i}\right|}+\sum_{i=1}^{2} s_{i} \operatorname{sign}\left(x-x_{i}\right) e^{-\left|x-x_{i}\right|}
$$

with $m_{1}=2.0, s_{1}=-1.0, m_{2}=-1.0, s_{2}=-0.5, x_{1}=-5.0$ and $x_{2}=5.0$. From Fig. 10, we can clearly see that two shockpeakons merge into one at $t \approx 3.5$, then the resulting shockpeakon gains a constant velocity and moves to the right. It illustrates the high resolution of the method to approximate the exact solutions, even in the presence of discontinuities.

\section{Conclusions}

We develop a splitting method for the DP equation, based on the OWENO reconstruction together with the Fourier pseudospectral discretization. A novel smoothness technique is presented to improve the resolution and the stability of OWENO-FPM. The formation of distinct nonlinear weights is discussed and analyzed in detail. Several coupling methods under this framework are provided to make comparison. Numerical examples show that OWENO-FPM have a comprehensively better performance in all examples. Moreover, we extend the framework to WENO-Z and the wavelet collocation method to explain flexibility and capability of the splitting strategy. Comparing with the DG method [17] or the spectral method [18], the proposed method can also achieve high order accuracy for the smooth solutions, but no limiter, filter or post-processing procedure is needed for the discontinuous solutions.

\section{Acknowledgements}

This work was supported by National Natural Science Foundation of China (Grant No. 91648204), National Key Research and Development Program of China 
(Grant No. 2016YFB0201301) and Science Challenge Project (Nos. JCKY2016212A502, TZ2016002).

\section{References}

[1] M. Procesi and A. Degasperis, Asymptotic integrability, in Symmetry and Perturbation Theory, World Scientific, Rome, 2000.

[2] Y. M. CHEN, S. H. SONG AND H. J. ZHU, Multi-symplectic methods for the Ito-type coupled KdV equation, Appl. Math. Comput., 218 (2012), pp. 5552-5561.

[3] X. Z. LIU, Y. JUN AND B. REN, New solutions from nonlocal symmetry of the generalized fifth order KdV equation, Chin. Phys. B, 24 (2015), 080202.

[4] A. Degasperis, D. D. Holm AND A. N. W. Hone, A new integrable equation with peakon solutions, Theor. Math. Phys., 133 (2002), pp. 1463-1474.

[5] A. PARKER, On the Camassa-Holm equation and a direct method of solution I. Bilinear form and solitary waves, Math. Phys. Eng. Sci., 460 (2004), pp. 2929-2957.

[6] Y. MATsuno, Multisoliton solutions of the Degasperis-Procesi equation and their peakon limit, Inverse Probl., 21 (2005), pp. 1553.

[7] B. F. Feng AND Y. LiU, An operator splitting method for the Degasperis-Procesi equation, J. Comput. Phys., 228 (2009), pp. 7805-7820.

[8] H. LundmaRK AND J. SZMigIELSKI, Multi-peakon solutions of the Degasperis-Procesi equation, Inverse Probl., 19 (2005), pp. 1241-1245.

[9] H. LundmaRK AND J. SZMIGIELSKI, Degasperis-Procesi peakons and the discrete cubic string, Int. Math. Res. Pap., 2005 (2005), pp. 53-116.

[10] A. Constantin And W. A. Strauss, Stability of peakons, Commun. Pure Appl. Math., 53 (2000), pp. 603-610.

[11] H. LUNDMARK, Formation and dynamics of shock waves in the Degasperis-Procesi equation, J. Nonlinear Sci., 17 (2007), pp. 169-198.

[12] G. M. COCLITE AND K. H. KARLSEN, On the well-posedness of the Degasperis-Procesi equation, J. Comput. Phys., 233 (2006), pp. 60-91.

[13] J. ESCHER, Y. LIU AND Z. YIN, Shock waves and blow-up phenomena for the periodic DegasperisProcesi equation, Indiana U. Math. J., 56 (2007), pp. 87-117.

[14] G. M. Coclite, K. H. Kenneth And N. H. Risebro, Numerical schemes for computing discontinuous solutions of the Degasperis-Procesi equation, IMA J. Numer. Anal., 28 (2008), pp. 80-105.

[15] Y. MiYATAKE AND T. Matsuo, Conservative finite difference schemes for the Degasperis-Procesi equation, J. Comput. Appl. Math., 236 (2012), pp. 3728-3740.

[16] C. H. YU AND T. W. H. SHEU, A dispersively accurate compact finite difference method for the Degasperis-Procesi equation, J. Comput. Phys., 236 (2013), pp. 493-512.

[17] Y. XU AND C. W. SHU, Local discontinuous Galerkin methods for the Degasperis-Procesi equation, Commun. Comput. Phys, 10 (2011), pp. 474-508.

[18] Y. XIA, Fourier spectral methods for Degasperis-Procesi equation with discontinuous solutions, J. Sci. Comput., 61 (2014), pp. 584-603.

[19] M. Z. SONG, X. QIAN AND S. SONG, Modified structure-preserving schemes for the DegasperisProcesi equation, Chin. Phys. Lett., 33 (2016), pp. 110202.

[20] G. S. JIANG AND C. W. SHU, Efficient implementation of weighted ENO schemes, J. Comput. Phys., 126 (1996), pp. 202-228. 
[21] X. D. LiU, S. Osher AND T. CHAN, Weighted essentially non-oscillatory schemes, J. Comput. Phys., 115 (1994), pp. 200-212.

[22] Y. XIA AND Y. XU, Weighted essentially non-oscillatory schemes for Degasperis-Procesi equation with discontinuous solutions, Ann. Math. Sci. Appl., 2 (2017), pp. 319-340.

[23] A. K. Henrick, T. D. Aslam AND J. M. POWERS, Mapped weighted essentially non-oscillatory schemes: achieving optimal order near critical points, J. Comput. Phys., 207 (2005), pp. 542-567.

[24] R. Borges, M. CARMONA, B. COSTA AND W. S. DON, An improved WENO scheme for hyperbolic conservation laws, J. Comput. Phys., 227 (2008), pp. 3191-3211.

[25] Y. Q. SHEN AND G. C. ZHA, Improvement of the WENO scheme smoothness estimator, Int. J. Numer. Meth. Fluids, 64 (2010), pp. 653-675.

[26] Y. HA, H. K. CHANG, Y. J. LEE AND J. YOON, An improved weighted essentially non-oscillatory scheme with a new smoothness indicator, J. Comput. Phys., 232 (2013), pp. 68-86.

[27] F. ACKer, R. B. D. R. BORges AND B. COsTA, An improved WENO-Z scheme, J. Comput. Phys., 313 (2016), pp. 726-753.

[28] D. L. Chai, Y. R. Li, Z. G. SUn AND G. XI, Analysis and improvement of weights in WENO schemes, J. Nat. Univ. Def. Technol., 38 (2016), pp. 39-45.

[29] J. WANG, Multi-symplectic Fourier Pseudospectral method for a higher order wave equation of KDV type, J. Comput. Math., 4 (2015), pp. 379-395.

[30] Y. M. CHEN, S. H. SONG AND H. J. ZHU, The multi-symplectic Fourier pseudospectral method for solving two-dimensional Hamiltonian PDEs, J. Comput. Appl. Math., 236 (2011), pp. 13541369.

[31] H. ZHU, L. TANG, S. SONG AND D. WANG, Symplectic wavelet collocation method for Hamiltonian wave equations, J. Comput. Phys., 229 (2010), pp. 2550-2572. 